\title{
The outer atmospheric layers of the early M dwarf Gliese 1
}

\author{
E. Lexen ${ }^{1}$, R. Wehrse ${ }^{1,2}$, J. Liebert ${ }^{3}$, and M. S. Bessell ${ }^{4}$ \\ ${ }^{1}$ Institut für Theoretische Astrophysik, Zentrum für Astronomie der Universität Heidelberg, Albert-Ueberle-Str. 2, \\ 69120 Heidelberg, Germany \\ e-mail: elexen;wehrse@ita.uni-heidelberg.de \\ 2 Interdisziplinäres Zentrum für Wissenschaftliches Rechnen (IWR), Im Neuenheimer Feld 368, 69120 Heidelberg, Germany \\ 3 Steward Observatory, Univ. of Arizona, Tucson, AZ 85721, USA \\ e-mail: liebert@as . arizona.edu \\ 4 RSAA, College of Science, The Australian National University, Cotter Road, Weston Creek ACT 2611, Australia \\ e-mail: bessell@mso.anu.edu.au
}

Received 6 May 2009 / Accepted 5 October 2009

\begin{abstract}
Using infrared and high-resolution optical observations of the M dwarf Gliese 1, we investigated the temperatures in the upper atmospheric layers of this star with low atmospheric activity. To fit the $\mathrm{H} \alpha$ and metal line profiles, the normal radiative equilibrium temperature decrease must be truncated at about $\log \tau_{1.2 \mu} \approx-1.9$ above which a steep chromospheric $(T \approx 4900 \mathrm{~K})$ rise must be imposed. Unfortunately, the position of the onset of the chromosphere depends to some extent on the temperature distribution in the inner parts of the photosphere. The chromosphere is just not sufficiently optically thick to be seen in the infrared up to $\approx 30 \mu \mathrm{m}$. The persistent strength of the TiO bands leads us to check for indications of surface inhomogeneities with negative results.
\end{abstract}

Key words. stars: atmospheres - stars: chromospheres - stars: late-type - stars: low-mass - stars: fundamental parameters

\section{Introduction}

M dwarfs are the most numerous stars (70\%) in the Milky Way. Even though they contribute about $40 \%$ to the total mass of the stellar contents, their atmospheres are still poorly understood due to the myriads of atomic and molecular lines, efficient convection that reaches very shallow layers, and strong chromospheres and coronae. Although model atmospheres have been used with increasing success to model the spectra of M dwarfs in recent years (Mould 1975, 1976; Allard 1990; Brett \& Plez 1993; Hauschildt et al. 1999; Gustafsson et al. 2003, 2008), uncertainties, particularly in the opacity data and in the modeling of nonradiative fluxes have up to now inhibited an accurate theoretical determination of the temperature distribution in the outer layers. Generally, the model atmosphere/synthetic spectra fits have emphasized the overall energy distributions and the molecular bands, with little attention paid to the profiles of atomic lines that are very sensitive to the outer layers. It is clear that a temperature inversion takes place at the top of the normal atmosphere in $\mathrm{M}$ dwarfs that show evidence of chromospheric activity, that is, emission lines of $\mathrm{H} \alpha$ and/or Ca II. However, many M dwarfs, especially those with kinematics that suggest they are very old, show little or no evidence of overt chromospheric activity.

Golimowski et al. (2004) present M band photometry for many dwarfs; however, only a few of the brightest M dwarfs have been observed spectroscopically in the $\mathrm{M}$ and $\mathrm{N}$ bands. In this paper, we report (in Sect. 2) observations of the M dwarf Gliese 1 (Gl 1) with the ISOPHOT spectrophotometer (Lemke et al. 1996) of the Infrared Space Observatory (ISO) in the wavelength range $2.5-12.5 \mu \mathrm{m}$ and the InfraRed Spectrograph (IRS) on the Spitzer Space Telescope (Houck et al. 2004) in the wavelength range $5.3-38 \mu \mathrm{m}$. These ISO and Spitzer observations are discussed in Sect. 3. In Sect. 2, medium and high-dispersion optical spectrophotometry of Gl 1 are also reported, while in Sect. 4 we attempt empirical determinations of the vertical temperature distribution of Gl 1 and show that the observed spectra do show evidence of chromospheric reversals. The summary discussion is given in Sect. 5 .

\section{Observations}

\subsection{Parameters of the observed star}

The star targeted for ISO and Spitzer observations provides ideal tests of the hypothesis that even inactive $\mathrm{M}$ dwarfs may retain chromospheric temperature reversals. Table 1 includes some relevant parameters and data for the target. It is noteworthy that Gl 1 is a dM star known to lack $\mathrm{H} \alpha$ emission, one sign of an active chromosphere. It shows old disk or halo kinematics. Eggen (1979) classified Gl 1 as old disk whereas Leggett (1992) and Leggett \& Hawkins (1988) classified Gl 1 as a halo star noting that it is subluminous in an $\mathrm{M}_{I} I-K$ color magnitude diagram, the signature of a metal-poor, Pop II M subdwarf. Gl 1 has an age of many Gyr, whether it is from the old disk or halo population. It has a slight metal deficiency in accordance with its space motion and it is probably massive enough to possess a small radiative core. Thus, it should be an example of a star in which a normal magnetic dynamo has had time to spin down the star. To our knowledge, direct measurements of the rotation $\left(v_{\sin _{i}}\right)$ are not available, since the star is generally too far south for the survey of Gliese stars by Stauffer \& Hartmann (1986). In the literature, the spectral type of Gl 1 ranges from M1.5V (Cincunegui \& Mauas 2004; Hawley et al. 1996) to M4V (Evans 1961; Gliese 1969). The spectral type would be close to M2V from $R-I$ and 
Table 1. Relevant parameters of Gl 1.

\begin{tabular}{lc}
\hline \hline Names & Gl 1, GJ 1, LHS 1 \\
& HIP 439, NSV 15017 \\
& HD 225213 \\
\hline FK5 coord. (ep = 2000 eq =2000) & $00^{\mathrm{h}} 05^{\mathrm{m}} 24.428^{\mathrm{s}}$ \\
$\alpha$ & $-37^{\circ} 21^{\prime} 26.50^{\prime \prime}$ \\
$\delta$ & $4.5 \mathrm{pc}$ \\
Distance & $\mathrm{M} 1.5$ \\
SpType & 10.25 \\
$M_{V}$ (Reid \& Hawley 2000) & 10.36 \\
$M_{V}$ (Reid & 6.33 \\
$M_{K}$ & 0.96 \\
$U-B$ & 1.45 \\
$B-V$ & 0.97 \\
$V-R$ & 2.12 \\
$V-I$ & 0.51 \\
$J-H$ & 0.22 \\
$H-K$ & \\
Space motions $\left[\mathrm{km} \mathrm{s}^{-1}\right]$ & -84 \\
$U$ & -99 \\
$V$ & -12 \\
$W$ & \\
\hline
\end{tabular}

Notes. the astronomical object database Simbad (2009) and Hawley et al. (1996), with colors from Reid \& Hawley (2000).

$V-I$ whereas $B-V$ is slightly bluer than an M2V star and implies M1V (Bessell 1991; Reid \& Hawley 2000). It is more physically realistic to use the red $R-I$ or $V-I$ color rather than the blue $B-V$ color (Bessell 1990). Using the TiO5 bandstrengths as suggested in Hawley et al. (1996) indicates it is close to M1.5V.

\subsection{ISOPHOT and IRS}

Observational data in the infrared were obtained from the Infrared Space Observatory (ISO) ISOPHOT (spectrophotometer) and the Infrared Spectrograph (IRS) on board the Spitzer Space Telescope in the wavelength range $5.3-38 \mu \mathrm{m}$ in December 2003. Details of the Spitzer science archive, pipeline upgrades and analysis tools are given in the IRS data handbook. The ISOPHOT (PHT40) spectrophotometry presented in this paper were obtained simultaneously in the two bands $2.5-5 \mu \mathrm{m}$ and $6-12 \mu \mathrm{m}$ on December 1996. The data were reduced using the ISOPHOT interactive analysis (PIA) package (Gabriel et al. 1997), a joint development by the ESA Astrophysics Division and the ISOPHOT Consortium. The data reduction procedure is fully described in the ISO Handbook (Laureijs et al. 2003). Figure 1 shows the good agreement between the observed ISOPHOT S and IRS fluxes in the overlapping wavelength region.

\subsection{Mt. Stromlo and Siding Spring spectrophotometry}

In order to investigate further the properties of this ISO and Spitzer target - in particular, to determine from line profiles whether they show any evidence of chromospheric activity or non-LTE effects - spectra were obtained in June 2007 with the Boller \& Chivens grating cross dispersed echelle spectrograph on the Nasmyth B focus on the $2.3 \mathrm{~m}$ reflector at Siding Spring Observatories. A $79 \mathrm{~g} / \mathrm{mm}$ echelle grating with dispersion $\lambda / 1000 \AA / \mathrm{mm}$ and a $2 \mathrm{kx} 2 \mathrm{k}$ Site CCD was used. The spectra have been divided by a smooth spectrum star to remove the telluric lines and the shape of the orders. The four wavelength regions contain the lines $\mathrm{Ca}$ II $\mathrm{H}$ and $\mathrm{K}$ (Fig. 2), Ca I (Fig. 3),

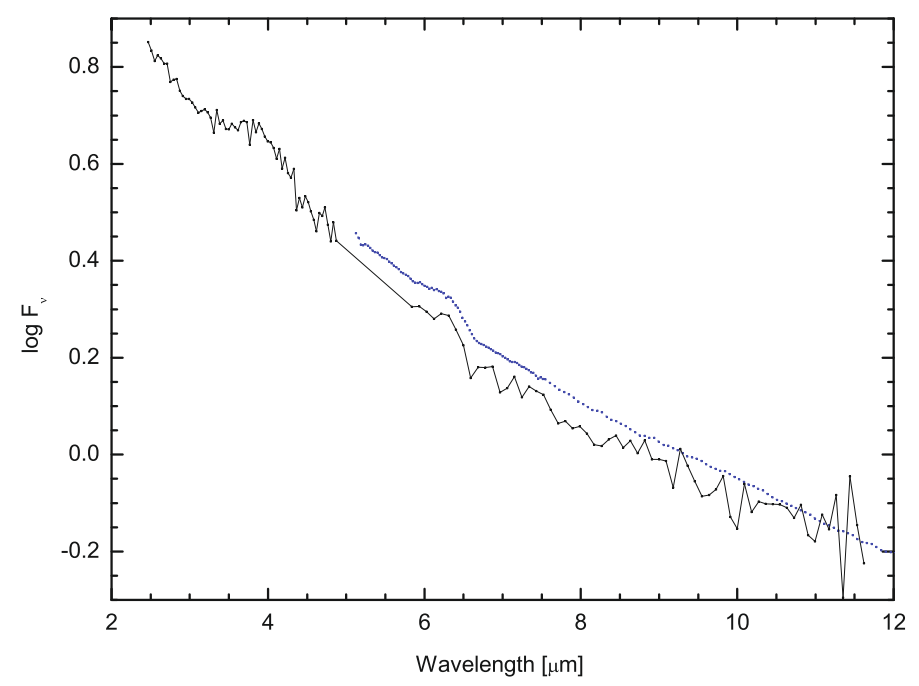

Fig. 1. ISOPHOT S (solid line) and IRS (dotted line) flux distributions for Gl 1.

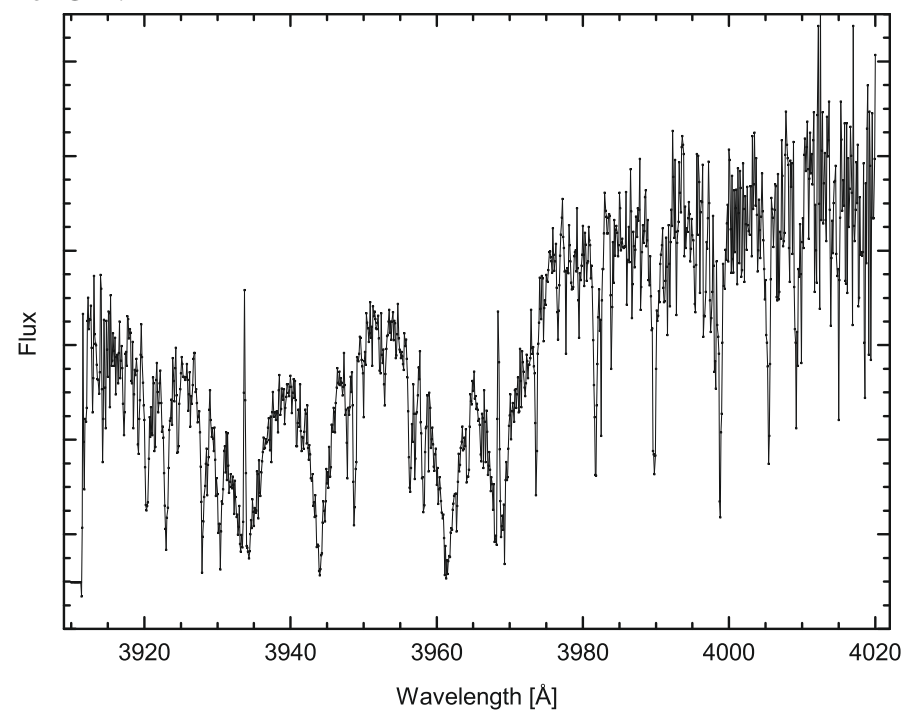

Fig. 2. The spectrum of G1 1 in the range of 3910-4020 $\AA$ around the $\mathrm{Ca}$ II H and $\mathrm{K}$ lines with a resolution of $100 \mathrm{~m} \AA$. Note the strong but very narrow emission in the cores of Ca II K (3933.7 ̊) and Ca II H (3968.5 А).

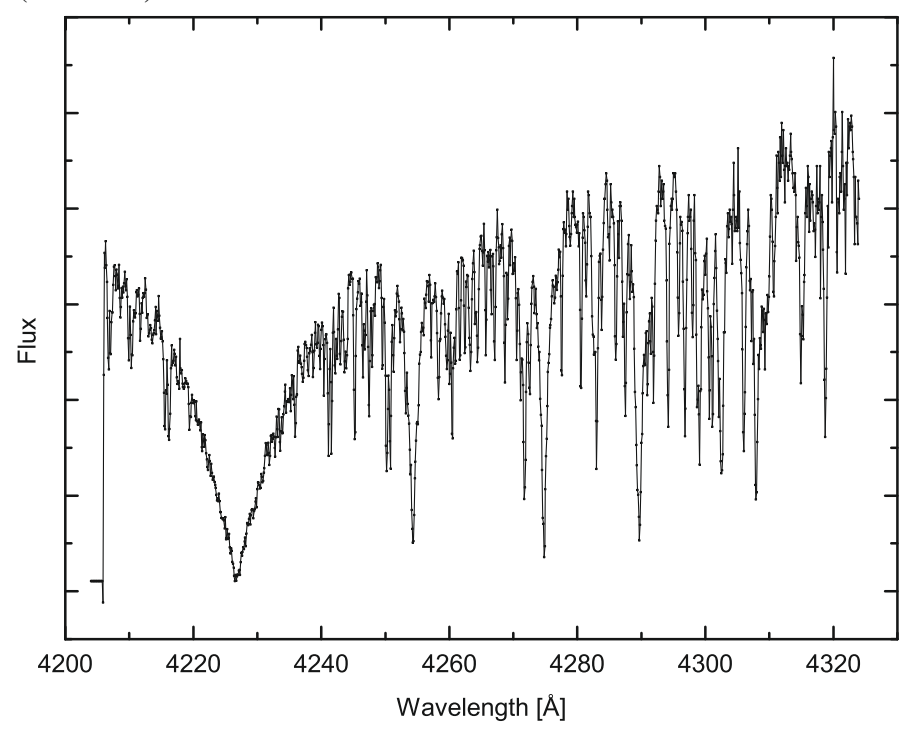

Fig. 3. The spectrum of G1 1 in the range of 4206-4324 $\AA$ around the Ca I line with a resolution of $100 \mathrm{~m} \AA$. 


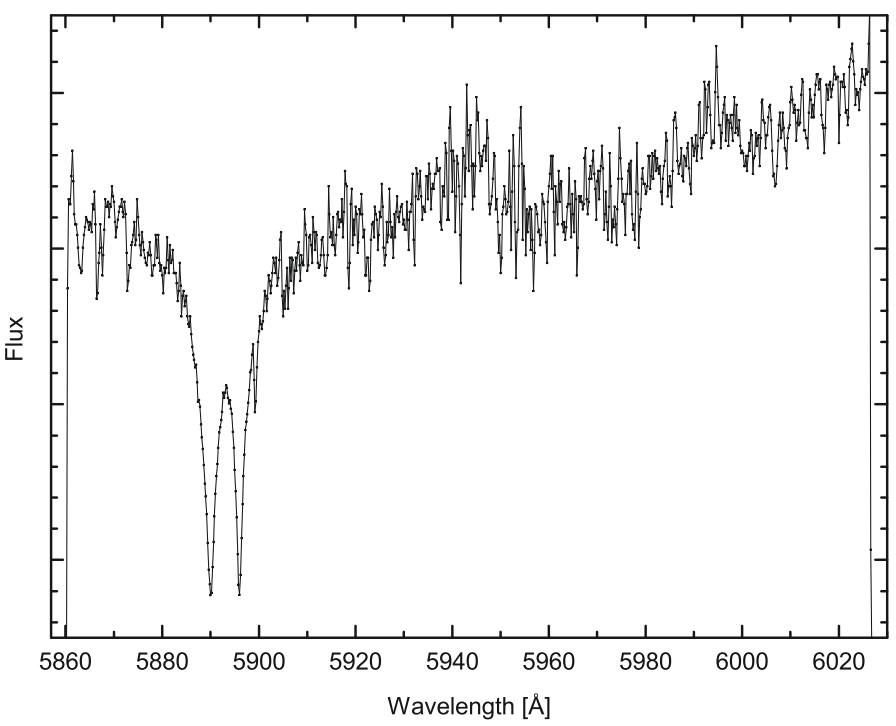

Fig. 4. The spectrum of Gl 1 in the range of 5860-6027 $\AA$ around the $\mathrm{Na} I \mathrm{D}_{2}$ and $\mathrm{D}_{1}$ lines with a resolution of $200 \mathrm{~m} \AA$.

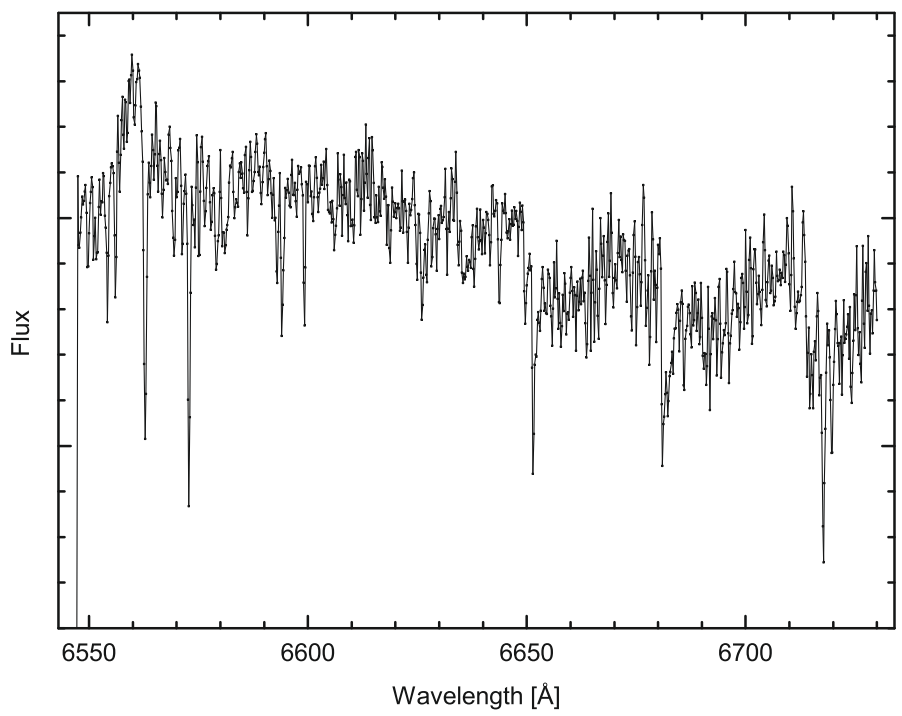

Fig. 5. The spectrum of Gl 1 in the range of 6546-6730 $\AA$ around the $\mathrm{H} \alpha$ line with a resolution of $200 \mathrm{~m} \AA$.

$\mathrm{Na} \mathrm{I} \mathrm{D}_{1}$ and $\mathrm{D}_{2}$ (Fig. 4) and $\mathrm{H} \alpha$ (Fig. 5). In addition, high resolution spectra were obtained with the coude echelle spectrograph on the $1.9 \mathrm{~m}$ reflector at Mount Stromlo Observatory prior to its loss in the 2003 fires. A $31.6 \mathrm{~g} / \mathrm{mm}$ echelle grating with the $81 \mathrm{~cm}$ camera and $2 \mathrm{kx} 4 \mathrm{k}$ Site CCD was used providing a resolution of $30 \mathrm{~mA}$. Different cross-disperser settings were required for blue and red wavelengths. These spectra have similarly been divided through by a smooth spectrum star to remove the telluric lines and to roughly flatten the spectrum. The spectra in each order was then flattened by hand so that the continuum was normalized to 1.0 .

\section{Infrared flux distribution}

In Fig. 10 is shown the observed ISOPHOT S (solid line) and IRS fluxes (dotted line) (shifted by an additional constant) compared with the distribution of a black body of $T_{\text {eff }}=3600 \mathrm{~K}$ and two calculated models with and without an additional cool

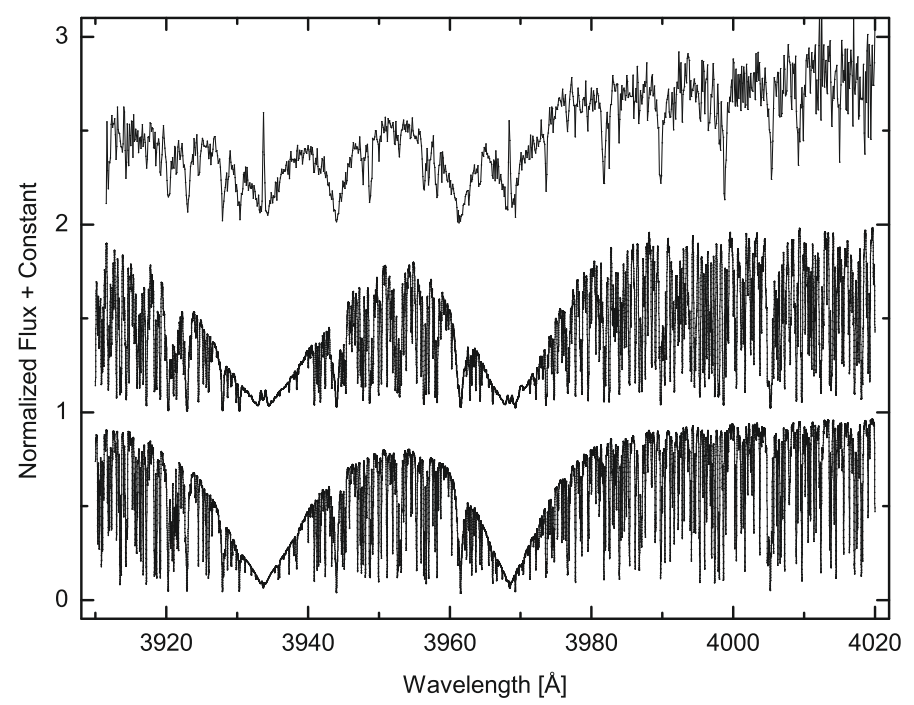

Fig. 6. The spectra of the stars G1 1 (M1.5), Arcturus (K1.5) and the Sun (G2) from top to bottom in the range of 3910-4020 $\AA$ around the $\mathrm{Ca}$ II $\mathrm{H}$ and $\mathrm{K}$ lines.

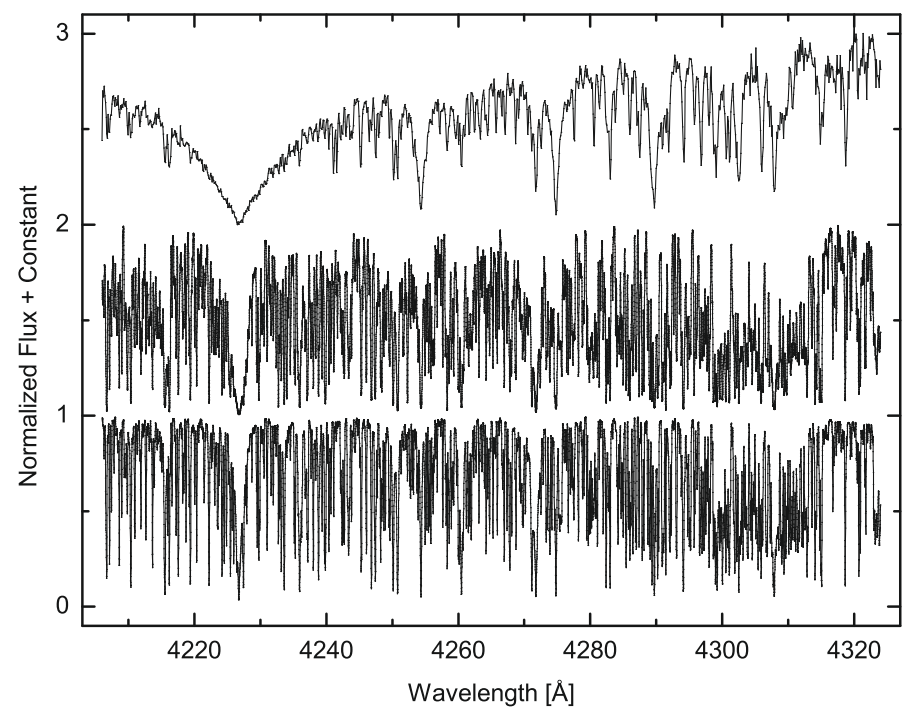

Fig. 7. The spectra of the stars Gl 1 (M1.5), Arcturus (K1.5) and the Sun (G2) from top to bottom in the range of 4206-4324 $\AA$ around the $\mathrm{Ca}$ I line.

chromosphere. The temperature stratification for the model with an additional component is shown in Fig. 18 (full line).

\section{Interpretation of the line profiles}

The obtained optical spectra of the M $1.5 \mathrm{~V}$ dwarf Gl 1 (Figs. 2-5) was compared with the spectra of the red giant Arcturus ( $\alpha$ Boo) (K1.5 III) and the Sun (G2V) (Figs. 6-9). The resolution of the spectra of Gl 1 are inferior to the others. The spectra of Arcturus (Hinkle et al. 2000) and the Sun (Kurucz 2005) are freely available on the web. For our purpose the spectrum of Arcturus has been corrected for negative values that were introduced by the observer for regions with poorly corrected telluric absorption features and for regions that were suspected to be affected by detector blemishes. Note that the very different appearances of the $\mathrm{Ca}$ II $\mathrm{H}$ and $\mathrm{K}$ lines of these objects reflect to a large extent the very different line blanketing. Furthermore, the emission components in the $\mathrm{H}$ and $\mathrm{K}$ lines (Fig. 6) reflect 


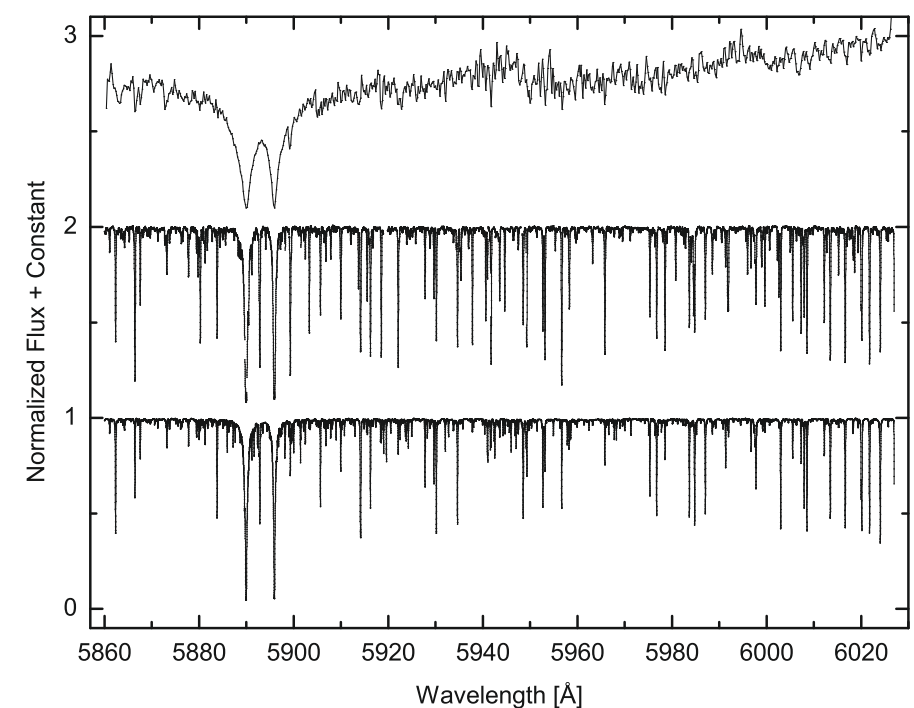

Fig. 8. The spectra of the stars G1 1 (M1.5), Arcturus (K1.5) and the Sun (G2) from top to bottom in the range of 5860-6027 $\AA$ around the $\mathrm{Na}$ I D lines.

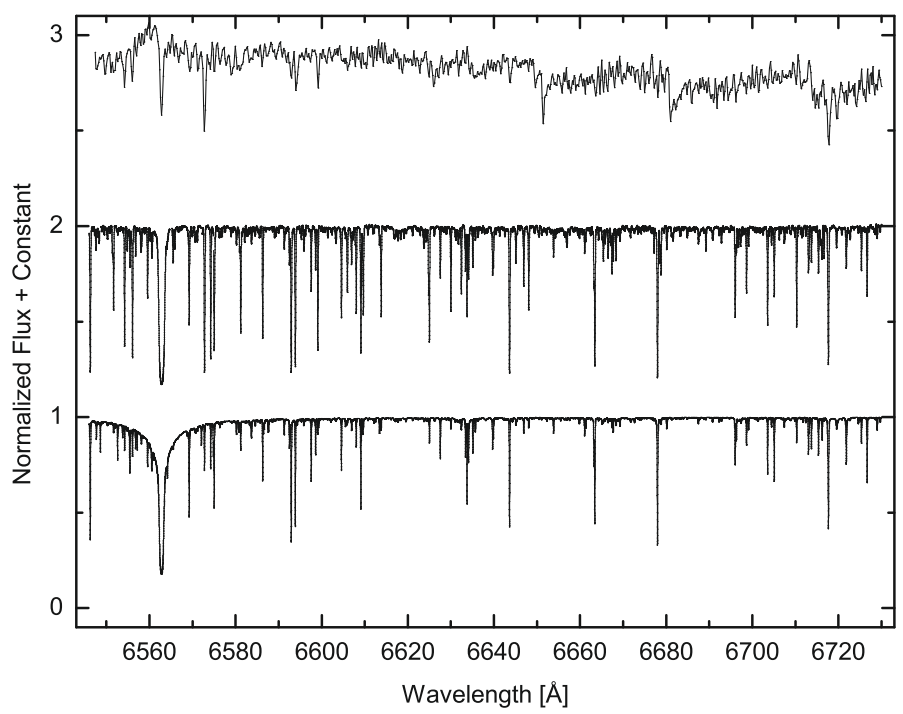

Fig. 9. The spectra of the stars G1 1 (M1.5), Arcturus (K1.5) and the Sun $(\mathrm{G} 2)$ from top to bottom in the range of 6546-6730 $\AA$ around the $\mathrm{H} \alpha$ line.

differences in the chromospheres. It is seen that in the dwarf Gl 1 very few lines and weak band heads can be identified and other lines form a quasi-continuum in the spectral range 6546-6730 (Fig. 9). A close look at the spectra of Gl 1 shows that several profiles of strong lines in the high dispersion optical and far-red spectra indicate deviations from the radiative equilibrium temperature distribution and from LTE level occupations in the top layers of the atmosphere: Fig. 2 shows evidence for reversals of the Ca II resonance line cores. The emission cores for the early objects require a reversal in the temperature in the top layers for at least some part of the photospheric disk.

A spectrum covering the $\mathrm{H} \alpha$ line is shown for Gl 1 in Fig. 5. Also seen in the spectral region of Fig. 5 is the strong $\mathrm{Ca} I$ line at $6572.795 \AA$, lines of Ti I 6554.298 and $6556.077 \AA$, and numerous weak $\mathrm{TiO}$ absorption features across the spectra. $\mathrm{H} \alpha$ is seen strongly in absorption in Gl 1 . With an equivalent width of $-0.39 \AA$ and $R-I=1.15$ (Robinson et al. 1990), Gl 1 falls near the bottom of the envelope of $\mathrm{M}$ dwarfs of similar color in Fig. 3

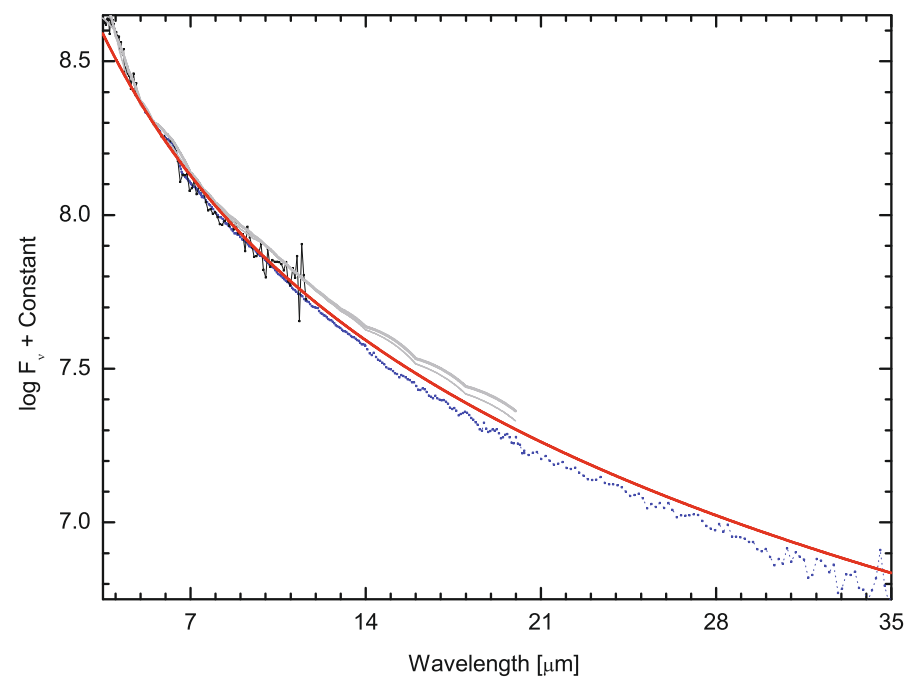

Fig. 10. Flux distributions for Gl 1 as shown in Fig. 1 in the range 3.5$35 \mu \mathrm{m}-$ and of synthetic spectra of models with (top gray line) and without (lower gray line) an additional cool chromosphere. A black body distribution of the same effective temperature is indicated by the solid line (center).

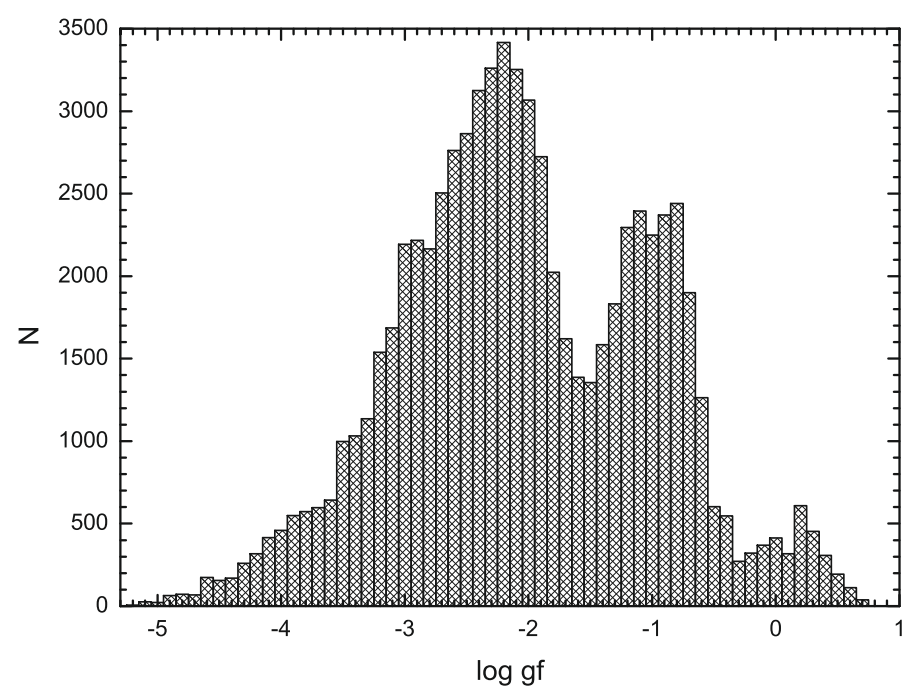

Fig. 11. Distribution of the numbers of titanium oxide lines (Plez 1998) used in the modeling of the spectrum in the wavelength range 6550$6580 \AA$ as a function of the gf-values of the lines. It is seen that there are essentially three peaks but the distributions are essentially continuous.

of Stauffer \& Hartmann (1986), Fig. 6 of Gizis et al. (2002) and Fig. 7 of Robinson et al. (1990).

The $n=2$ lower level of hydrogen has an excitation potential of $10.2 \mathrm{eV}$. For a simple atmosphere in radiative equilibrium at the indicated temperature near $3600 \mathrm{~K}$, the corresponding Boltzmann factor would be smaller than $10^{-17}$ and no $\mathrm{H} \alpha$ absorption should appear. Instead, it is necessary to assume that the line is actually formed in hotter, outer layers and is subject to strong NLTE effects that reduce the source function. These findings are in accordance with the calculations of Cram \& Mullan (1979), Houdebine et al. (1995) and Short \& Doyle (1997) and others; these show that the first effect of a weak chromospheric reversal in the outer atmospheric layers is to produce a pure absorption line. As the reversal becomes stronger, the line gets weaker, then finally goes into emission. Empirical evidence in favor of this interpretation is discussed by Stauffer \& Hartmann (1986). The strength of the absorption actually indicates a weak 


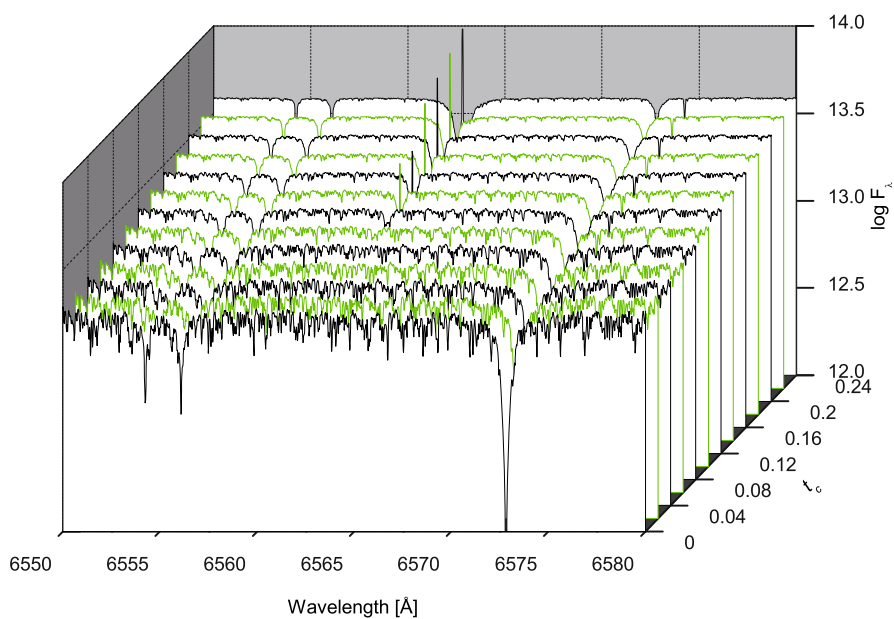

Fig. 12. Model series with gray temperature stratification for $T_{\text {eff }}=$ $3600 \mathrm{~K}$ and $\log g=5$ and chromospheric temperature rises for $\delta=0.01$, $\tau_{0}=0.1$ and $\Delta t_{\mathrm{c}}=0.02 t_{\mathrm{c}} \in[0.0-0.24]$ in ascending order. For the definitions of parameters see text. The chemical composition in the models is assumed to be solar except for $\mathrm{Ca}$ and Fe reduced by $1 \mathrm{dex}$ and $\mathrm{Ti}$ reduced by 0.3 dex.

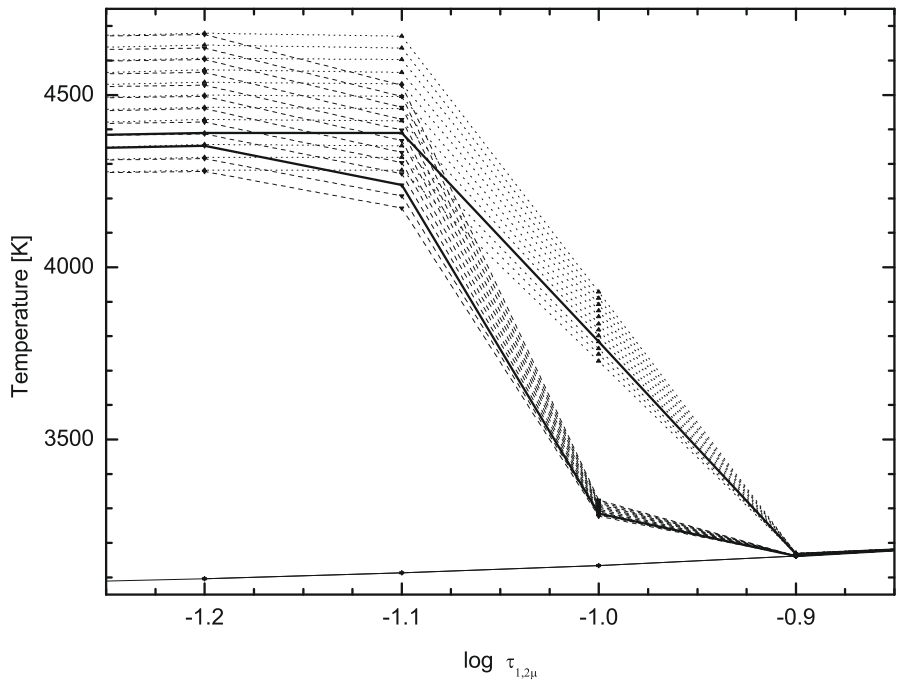

Fig. 13. Temperature as a function of the optical depth at $1.2 \mu$ with one data point per 0.1 in $\log \tau_{1.2 \mu}$. Stratifications for the fluxes plotted in Fig. 12 with $\delta=0.01$ and $\tau_{0}=0.1$ (dotted lines) and $\tau_{0}=0.09$ (dashed lines). The temperature rise starts at $\log \tau_{1.2 \mu}=-0.9$ and $T \approx 3160 \mathrm{~K}$. Temperature stratifications with $t_{\mathrm{c}}=0.18, \tau_{0}=0.1$ and $t_{\mathrm{c}}=0.175, \tau_{0}=$ 0.09 (full lines) for the best model of this series are plotted in Figs. 14 and 15 . For the meaning of the parameters modeling the chromospheric temperature rise see text.

chromosphere compared with stars of similar colors (Fig. 14) in the Stauffer \& Hartmann (1986) sample, because the absorptions are stronger than the average. Cram \& Mullan (1979) calculated for an atmospheric $T_{\text {eff }}$ of $3500 \mathrm{~K}$ that the maximum absorption equivalent width would be $-0.69 \AA$, of which only $-0.08 \AA$ is caused by the normal photosphere. Gl 1 has an equivalent width of $-0.39 \AA$ (Robinson et al. 1990; Panagi \& Mathioudakis 1993). The neighboring Ca I resonance line (6572 $\AA$ ) is deeper than expected from a radiative equilibrium atmosphere. We suspect therefore that it is also formed in "scattering mode". It is very narrow, implying that the microturbulence plus rotational velocity is quite low. The spectra certainly require a full NLTE

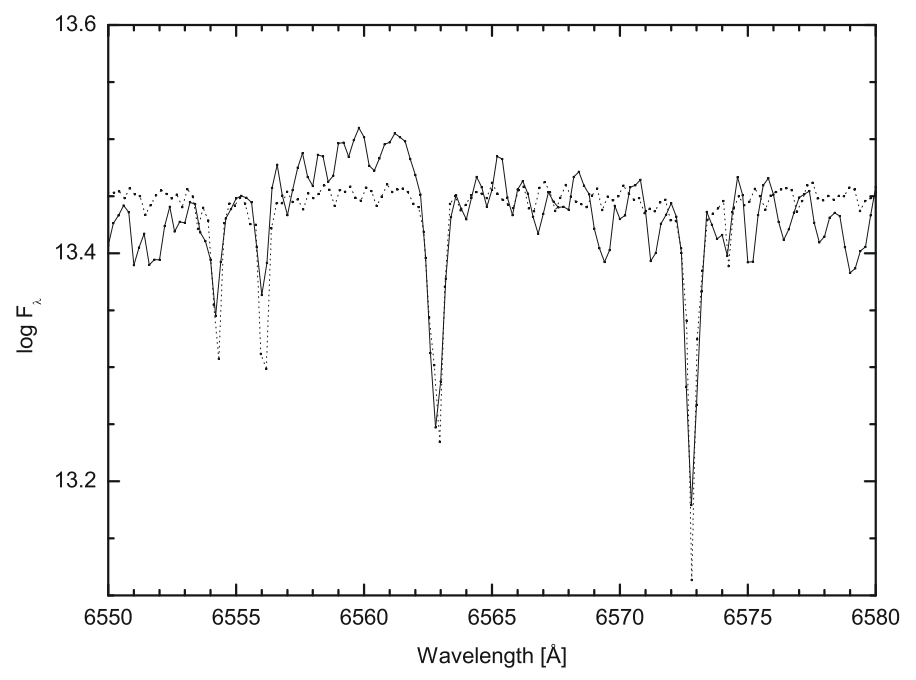

Fig. 14. Comparison of high resolution observations of Gl 1 (full line) and synthetic spectra calculated with an additional cool chromosphere with parameters $t_{\mathrm{c}}=0.175, \tau_{0}=0.09$ as shown in Fig. 13 (dashed line).

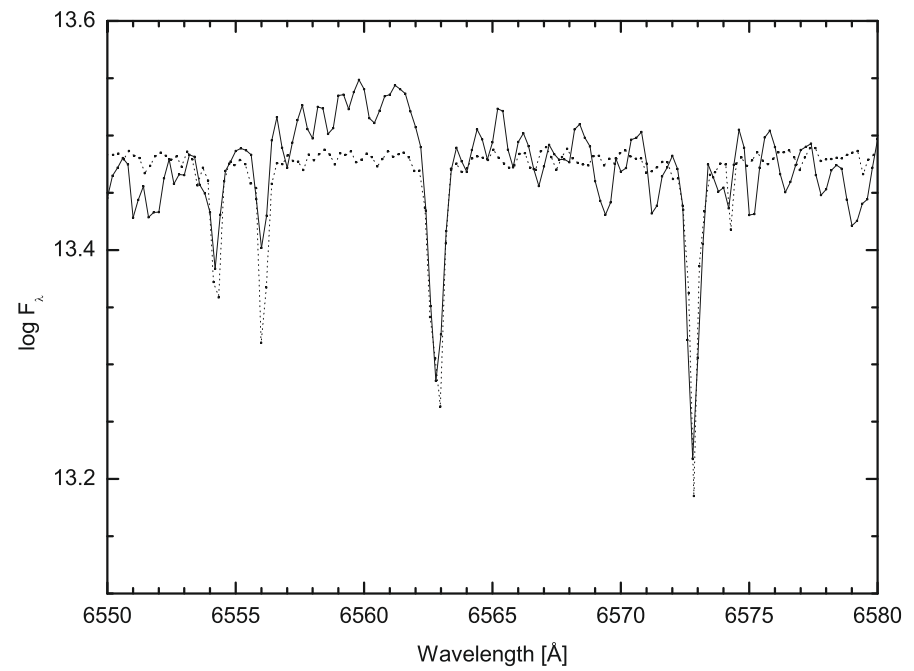

Fig. 15. Same as Fig. 14 but for parameters $t_{\mathrm{c}}=0.18$ and $\tau_{0}=0.1$.

analysis. Unfortunately, a corresponding program is presently not available to us.

However, in order to see qualitatively the consequences of a chromospheric temperature rise on the spectrum in the $\mathrm{H} \alpha$ region, we used the program of Wehrse (1981) that can treat 2-level atoms, i.e. we split the extinction coefficient into an absorption and a scattering fraction and describe the ratio of absorption to extinction coefficient by the parameter $\epsilon$ (cf. Jefferies 1968; Mihalas 1978). We include the TiO line list of Plez (1998) and the atomic lines Ti I (6554.238 and 6556.077 $\AA$ ), Ca I $(6572.795 \AA)$ and Fe I (6574.254 $\AA$ ) and assume for all lines $\epsilon=10^{-4}$, i.e. nearly complete scattering mode. A statistical evaluation of the transition probability $\times$ statistical weight plane for the used TiO lines is shown in Fig. 11. Due to the uncertainties we choose $T_{\text {eff }}=3600 \mathrm{~K}$, however from $V-I$ a more reasonable value would be closer to $3500 \mathrm{~K}$. First we calculated classical models with gray temperature stratification (Fig. 12), hereafter we included convection by using the extrapolated temperature stratification from MARCS. In order to model a chromospheric temperature inversion we add to the temperature distribution 


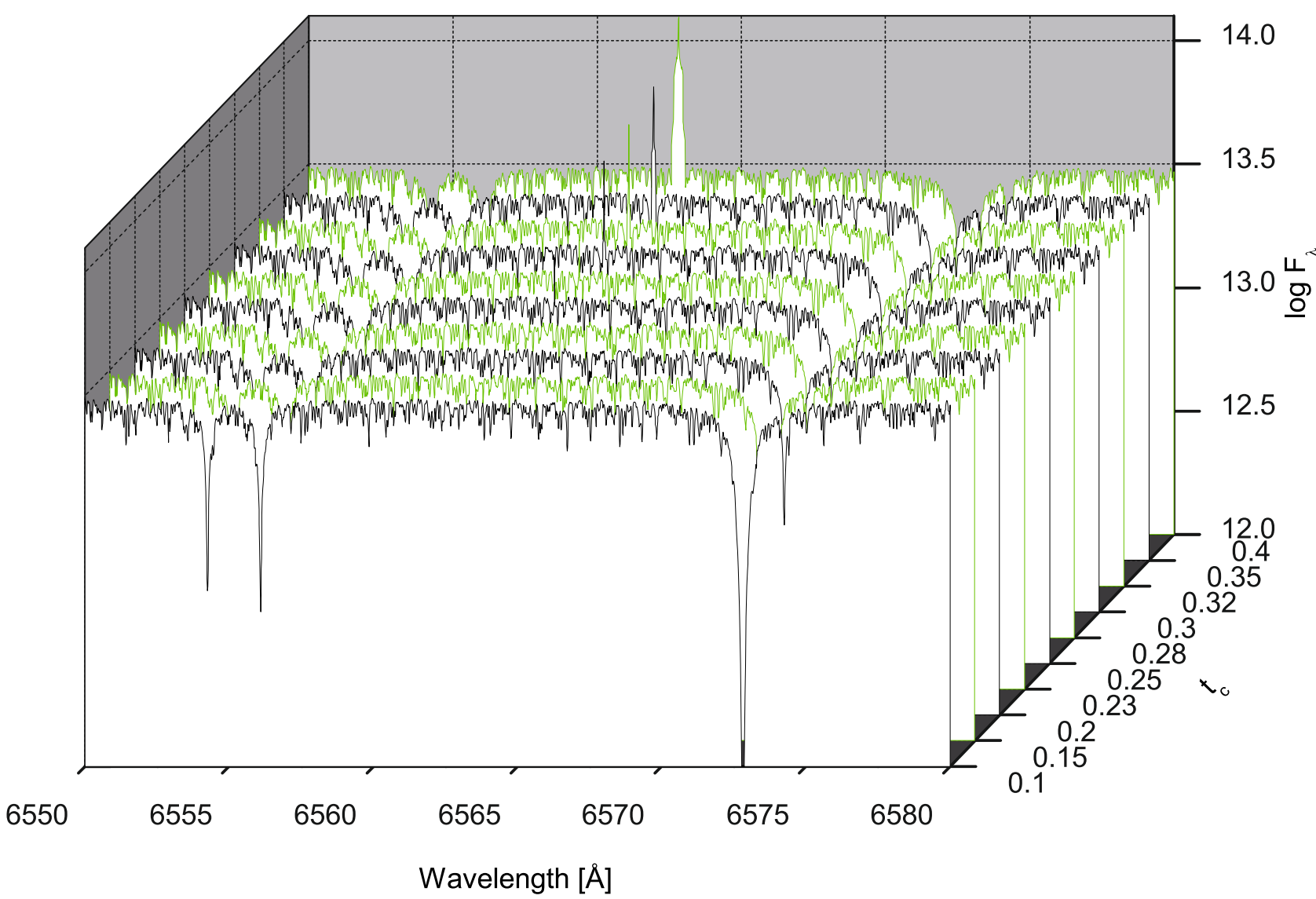

Fig. 16. Synthetic spectra calculated with an additional cool chromosphere including convection with $T_{\text {eff }}=3600 \mathrm{~K}$ and $\log g=5$. Chromospheric temperature rise is shown for parameters $\delta=0.0001, \tau_{0}=0.001$ with temperature stratification $t_{\mathrm{c}}=[0.10 ; 0.15 ; 0.20 ; 0.23 ; 0.25 ; 0.28 ; 0.30 ; 0.32$; 0.35; 0.40] (from front to back). The chemical composition in the models is assumed to be solar except for Ca and Fe reduced by 1 dex. Note the lack of $\mathrm{H} \alpha$ absorption in the series of spectra.

$t_{\mathrm{c}}\left(1-\tanh \left(\frac{\tau_{1.2 \mu}-\tau_{0}}{\delta}\right)\right)$. By varying the parameters $t_{\mathrm{c}}, \tau_{0}$ and $\delta$ we can now modify the height, the abruptness and the inflection point of the chromospheric temperature rise. Assumed gray temperature stratification with various chromospheric distributions are shown in Fig. 13. The best model fits are compared with the observations in Figs. 14 and 15.

From these comparisons we see that:

(i) without an outer temperature rise the molecular lines are reasonably well reproduced. A large fraction of the discrepancies most probably result from uncertainties in the $\mathrm{TiO}$ line data as comparisons with Kurucz's and Joergensen's list have shown, but the atomic lines do not fit at all; in particular, $\mathrm{H} \alpha$ absorption is completely absent, as expected from the cited earlier papers;

(ii) with an increasing temperature rise, the atomic lines fit reasonably well but now the molecular features are too weak;

(iii) the predicted $\mathrm{H} \alpha$ line is completely absent without an outer temperature rise. With a temperature rise, $\mathrm{H} \alpha$ starts with an increasing absorption component, finally superimposed with a very narrow emission core that is not observed. We therefore modified the temperature distribution in various ways to avoid the emission core without losing the absorption part.

In order to include convection, we use an extrapolated temperature stratification from MARCS. The chemical composition for the calculated models is assumed to be solar except for $\mathrm{Ca}$ and Fe which were reduced by 1 dex. In these models it can be shown that synthetic spectra in agreement with observations can only be reached by starting the chromospheric temperature rise at the specific optical depth that was empirically determined. Calculated fluxes are shown for parameters $\delta=0.0001$ and $\tau_{0}=0.001$ that indicate the chromospheric temperature rise at an optical depth $\log \tau_{1.2 \mu} \approx-2.5$ for different temperature stratifications (Fig. 16). In these series the $\mathrm{H} \alpha$ absorption is completely absent. Varying the parameters $\delta=0.001$ and $\tau_{0}=0.01$ for starting the beginning of the chromospheric temperature rise at an optical depth $\log \tau_{1.2 \mu} \approx-1.9$ leads to the models shown in Fig. 17. The temperature stratification for this model series is shown in Fig. 18. The best model fit of this series is compared with high resolution observations of Gl 1 (Fig. 19). In the last series, we use the parameters $\delta=0.01$ and $\tau_{0}=0.07$ so the beginning of the chromospheric temperature rise starts at an optical depth $\log \tau_{1.2 \mu} \approx 1.9 \log \tau_{1.2 \mu} \approx-1$ (Fig. 20). As expected, the molecular features are too weak and the $\mathrm{H} \alpha$ line can't be modeled in absorption without an emission core in this series. It seems impossible to get a comparable fit for a model in which only the abundances of $\alpha$ elements relative to iron are enhanced.

\section{Discussion}

It had been the original plan (Wehrse et al. 1997) to detect as a consequence of the strong increase of the $\mathrm{H}^{-}$absorption 


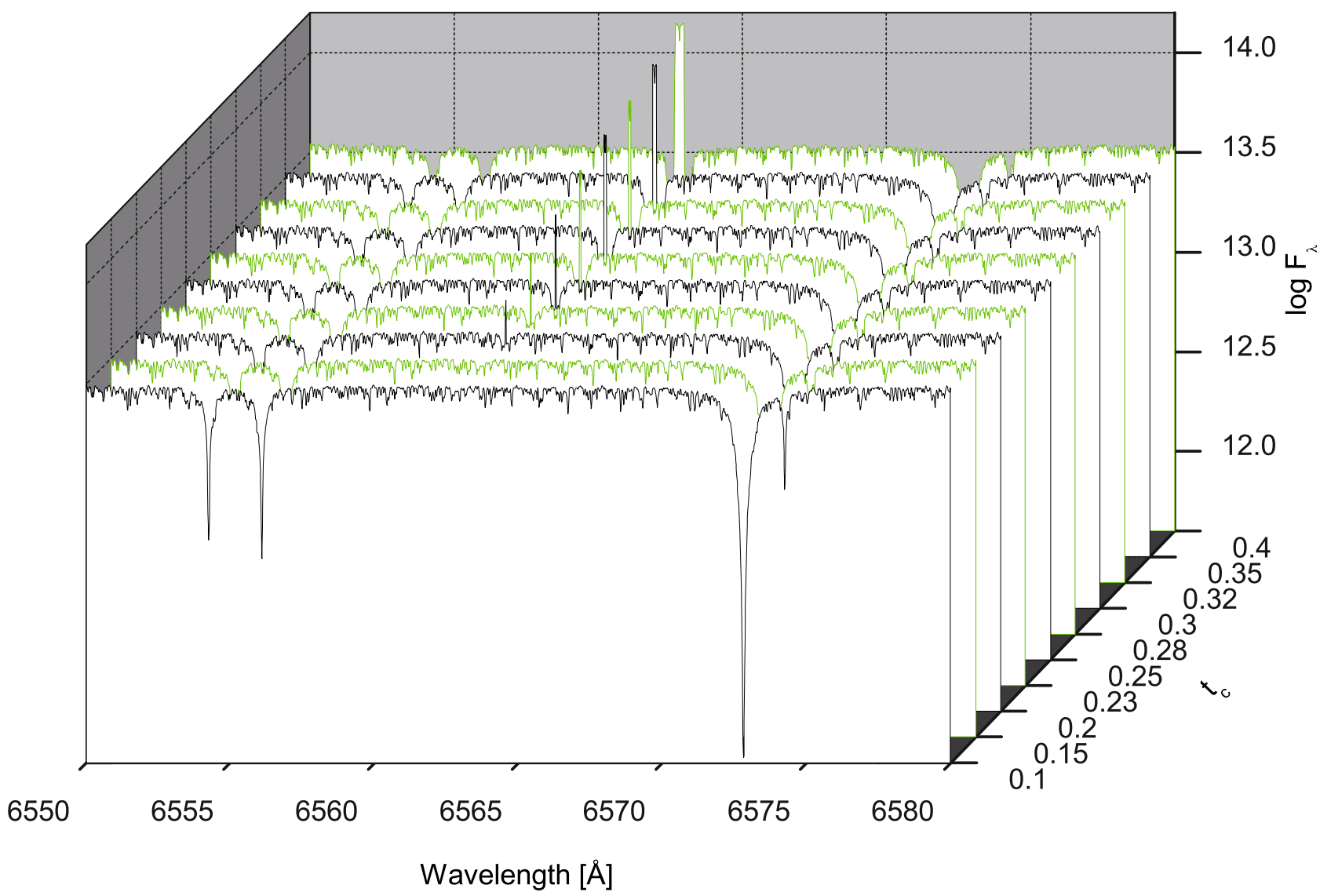

Fig. 17. Same as Fig. 16 but for parameters $\delta=0.001$ and $\tau_{0}=0.01$. The temperature stratification for this model series is shown in Fig. 18. Note that weak $\mathrm{H} \alpha$ absorption is evident in some spectra but with a strong emission core.

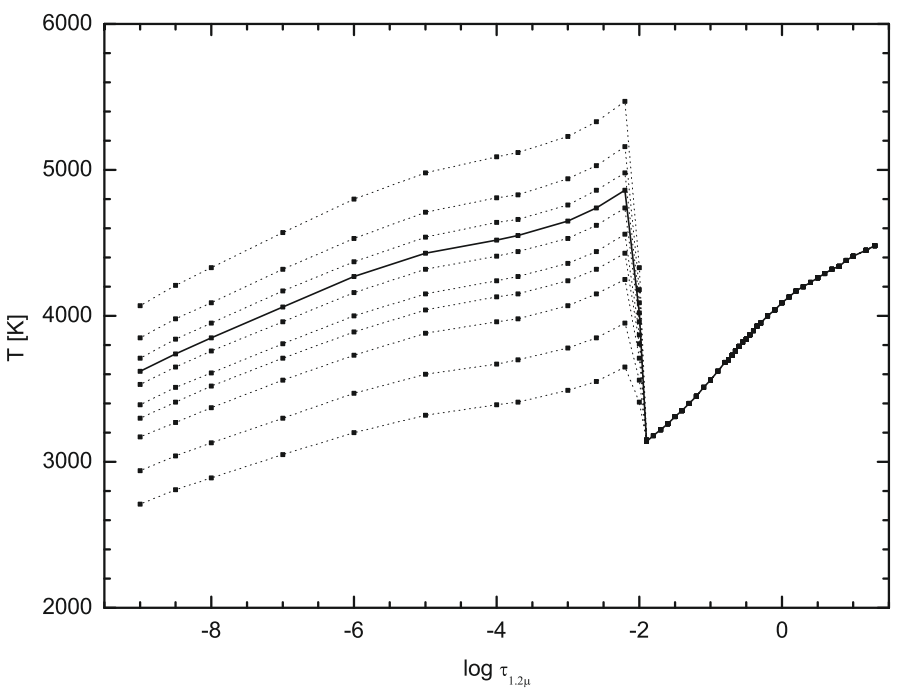

Fig. 18. Temperature as a function of the optical depth at $1.2 \mu$ for the fluxes plotted in Fig. 17. The temperature rise starts at $\log \tau_{1.2 \mu}=-1.9$ and $T=3140 \mathrm{~K}$ the temperature stratification with $t_{\mathrm{c}}=0.3$ (full line) for the best model of this series is plotted in Fig. 19.

coefficient with wavelength - the chromosphere of M dwarfs in the IR range accessible to the ISO and Spitzer space crafts, and to use these observations to model empirically the temperature distributions in the very outer layers of these stars. This would

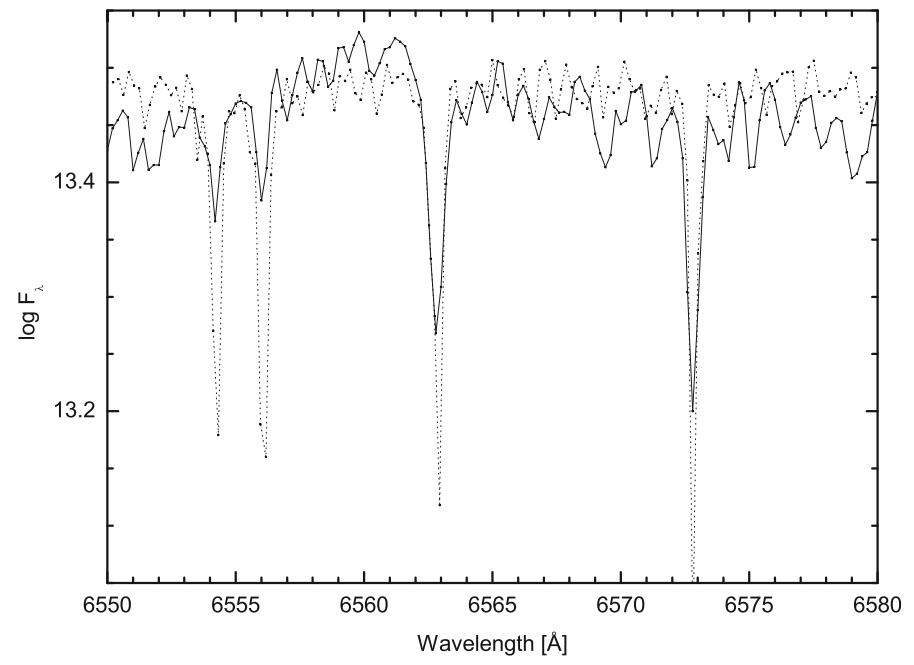

Fig. 19. Comparison of high resolution observations of Gl 1 (full line) and best model fit from Fig. 17. It is calculated with an additional cool chromosphere including convection with parameters $t_{\mathrm{c}}=0.3$ (temperature stratification as shown in Fig. 18), $\tau_{0}=0.01$ and $\delta=0.001$ (dashed line).

allow the study of the outer radiative boundary condition needed in the construction of chromospheric and interior models.

Unfortunately, a flux excess increasing to longer wavelengths is not seen for Gl 1 up to about $35 \mu \mathrm{m}$. The reason for this can be seen from Fig. 21. In spite of a strong increase with 


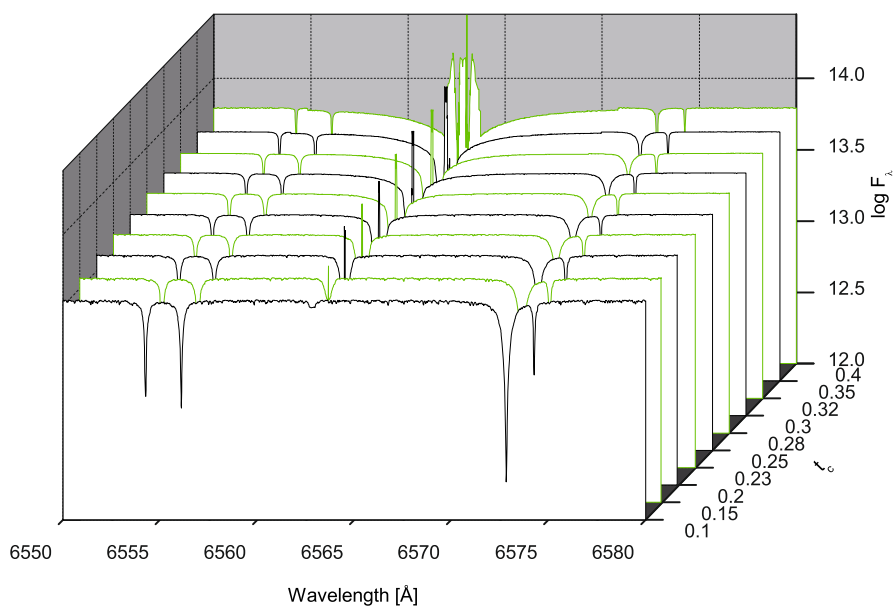

Fig. 20. Same as Fig. 16 but for parameters $\delta=0.01$ and $\tau_{0}=0.07$. Note the strong $\mathrm{H} \alpha$ absorption accompanied by an emission core in many of the spectra while the molecular lines are always too weak.

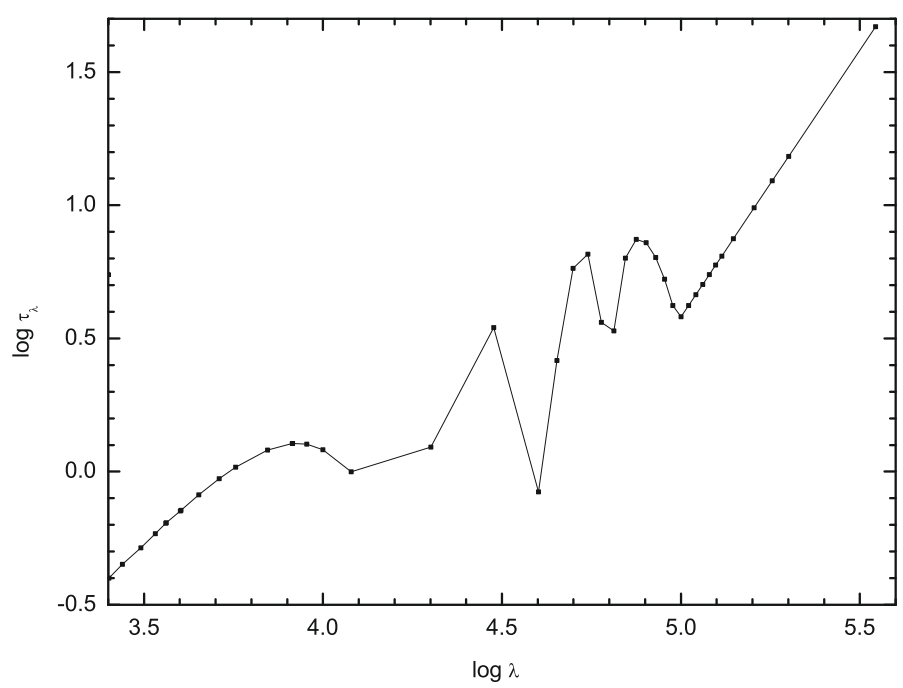

Fig. 21. Essential run of the optical depth of the layer $\tau_{1.2 \mu}=1$ with wavelength for the same parameters as the model plotted in Fig. 15. Note that continuous and water absorption coefficients at wavelengths indicated by the dots are involved.

wavelength, $\tau_{\lambda}\left(\tau_{\text {std }}=10^{-1.9}\right)$ does yet not reach increase unity, i.e. we see essentially only the layers around the temperature minimum! This is most probably the reason for the flux decrease that is steeper than that of a black body (Fig. 10). With increasing wavelengths, layers of lower and lower temperature are seen. Since we have not modelled the flux of Gl 1 consistently from the UV to the IR (e.g. with respect to the inclusion of all relevant lines), we cannot give accurate total acoustic plus magnetic fluxes that are dissipated in the chromosphere but our models indicate an appreciable fraction (up to about 25\%) of the total flux entering the atmosphere must be of non-radiative nature. If this is confirmed, these fluxes will have severe consequences for the temperature stratification for layers below the chromosphere also and must consistently be taken into account in future modeling. This would imply that the effective temperature, the gravity, and the chemical composition are not sufficient to determine the atmospheres of $\mathrm{M}$ dwarf stars uniquely. The combination of the strong $\mathrm{H} \alpha$ and $\mathrm{Ca}$ I absorption, the Ca II emission reversals, and the persistent strength of the TiO bands lead us to check for indications of surface inhomogeneities. We assume that the atmosphere is divided into two components (e.g. Giampapa 1985). Some fraction of the surface is spotted with chromospheric activity, but the majority of the surface probably lacks activity. Just like Houdebine et al. (1995) we try to merge our calculated atmospheres. This method did not provide any significant indications of surface inhomogeneities.

The element abundances given in Sect. 4 have been determined iteratively starting from solar values and seemed to give the best fits to our spectra. In view of the complicated dependencies of the line strengths and profiles on the details of the temperature stratification and of the chemical composition (cf. Allard \& Hauschildt 1995; see also Wehrse 1985, for an analogue discussion for M giants), we cannot, however, exclude that a fit to the full spectrum may result in a somewhat different composition, e.g. in an enhanced abundance ratio of the $\alpha$ elements to iron. A thorough quantitative error analysis on the basis of well calibrated high dispersion spectra extending from the visible to the infrared range is certainly an urgent need for cool dwarf stars such as Gl 1.

In summary, we have analyzed high resolution optical and low resolution infra-red spectra of the early $M$ dwarf Gliese 1 in order to study the temperature structure of the upper photospheric layers. From the $\mathrm{H} \alpha$ and adjacent metal line profiles it is found that there should be a temperature reversal at about $\tau_{1.2 \mu} \approx 0.01$ and an outer temperature of $\sim 4900 \mathrm{~K}$. There are no indications of horizontal inhomogeneities or of chromospheric flux enhancements in the IR up to $\lambda \approx 30 \mu \mathrm{m}$. It was beyond the scope of this paper to perform a full and consistent abundance analysis, the modeling of the metal lines requiered decreased abundances for $\mathrm{Ca}, \mathrm{Fe}$ and $\mathrm{Ti}$.

\section{References}

Allard, F. 1990, Ph.D. Thesis, Ruprecht Karls Univ Heidelberg Allard, F., \& Hauschildt, P. H. 1995, ApJ, 445, 433 Bessell, M. S. 1990, A\&AS., 83, 357

Bessell, M. S. 1991, AJ, 101, 662

Brett, J. M., \& Plez, B. 1993, Proc. Astron. Soc. Australia, 10, 250

Cincunegui, C., \& Mauas, P. J. D. 2004, A\&A, 414, 699

Cram, L. E., \& Mullan, D. J. 1979, ApJ, 234, 579

Eggen, O. J. 1979, ApJ, 230, 786

Evans, D. S. 1961, Royal Greenwich Observatory Bulletin, 48, 389

Gabriel, C., Acosta-Pulido, J., Heinrichsen, I., Morris, H., \& Tai, W.-M. 1997, in Astronomical Data Analysis Software and Systems VI, ed. G. Hunt, \& H. Payne, ASP, 125, 108

Giampapa, M. S. 1985, ApJ, 299, 781

Gizis, J. E., Reid, I. N., \& Hawley, S. L. 2002, AJ, 123, 3356

Gliese, W. 1969, Veröffentlichungen des Astronomischen Rechen-Instituts Heidelberg, 22, 1

Golimowski, D. A., Leggett, S. K., Marley, M. S., et al. 2004, AJ, 127, 3516

Gustafsson, B., Edvardsson, B., Eriksson, K., et al. 2003, in Stellar Atmosphere

Modeling, ed. I. Hubeny, D. Mihalas, \& K. Werner, ASP Conf. Ser., 288, 331 Gustafsson, B., Edvardsson, B., Eriksson, K., et al. 2008, A\&A, 486, 951

Hauschildt, P. H., Allard, F., \& Baron, E. 1999, ApJ, 512, 377

Hawley, S. L., Gizis, J. E., \& Reid, I. N. 1996, AJ, 112, 2799

Hinkle, K., Wallace, L., Valenti, J., \& Harmer, D. 2000, Visible and Near Infrared Atlas of the Arcturus Spectrum 3727-9300 A, ed. K. Hinkle, L. Wallace, J. Valenti, D. Harmer

Houck, J. R., Roellig, T. L., van Cleve, J., et al. 2004, ApJ, 154, 18

Houdebine, E. R., Doyle, J. G., \& Koscielecki, M. 1995, A\&A, 294, 773

Jefferies, J. T. 1968, Spectral line formation, ed. J. T. Jefferies

Kurucz, R. L. 2005, Mem. Soc. Astron. Ital. Supp., 8, 189

Laureijs, R. J., Klaas, U., Richards, P. J., Schulz, B., \& Abraham, P. 2003,

The ISO Handbook, Vol. IV - PHT - The Imaging Photo-Polarimeter, ed.

T.G. Mueller, J.A.D.L. Blommaert, \& P. Garcia-Lario., ESA SP-1262

Leggett, S. K. 1992, ApJ, 82, 351

Leggett, S. K., \& Hawkins, M. R. S. 1988, MNRAS, 234, 1065

Lemke, D., Klaas, U., Abolins, J., et al. 1996, A\&A, 315, L64 
E. Lexen et al.: The outer atmospheric layers of the early M dwarf Gliese 1

Mihalas, D. 1978, Stellar atmospheres, 2nd edition, ed. J. Hevelius

Mould, J. R. 1975, A\&A, 38, 283

Mould, J. R. 1976, A\&A, 48, 443

Panagi, P. M., \& Mathioudakis, M. 1993, A\&AS, 100, 343

Plez, B. 1998, A\&A, 337, 495

Reid, N., \& Hawley, S. L., 2000, New light on dark stars: red dwarfs, low mass stars, brown dwarfs

Robinson, R. D., Cram, L. E., \& Giampapa, M. S. 1990, ApJS, 74, 891

Short, C. I., \& Doyle, J. G. 1997, A\&A, 326, 287
Stauffer, J. R., \& Hartmann, L. W. 1986, ApJS, 61, 531

Wehrse, R. 1981, MNRAS, 195, 553

Wehrse, R. 1985, in Cool Stars with Excesses of Heavy Elements, Proceedings of the Strasbourg Observatory Colloquium, Universite de Strasbourg I, France, July 3-6, 1984 (Dordrecht: D. Reidel Publishing Co.) ed. M. Jaschek, \& P. C. Keenan, Astrophysics and Space Science Library, 114, 293

Wehrse, R., Rosenau, P., Suvernev, A., Liebert, J., \& Leinert, C. 1997, in The first ISO workshop on Analytical Spectroscopy, ed. A. M. Heras, K. Leech, N. R. Trams, \& M. Perry, ESA SP, 419, 309 